\title{
Web Pages that Reproduce Themselves by JavaScript
}

\author{
Yasusi Kanada \\ Central Research Laboratory, Hitachi Ltd. \\ Higashi-Koigakubo 1-280, Kokubunji, Tokyo 185, Japan
}

\begin{abstract}
A JavaScript program in a Web page can clear the page content including the program itself and generate new content. The program can generate exactly the same content including the program itself. This means that a Web page can reproduce itself by JavaScript program that is included in the page. Although exact reproduction is useless, inexact reproduction, which transform part of the content, is usable for more practical purpose. For example, Web pages that change its view from outline mode to detail mode by clicking a button in the page can be implemented using this method. This method can also applicable to other types of documents, such as SGML or XML, if the document may contain self-reproductive program. Another method for reproducing Web pages without reproducing programs is also mentioned. Reproductive Web pages partially but really work on Netscape Navigator.
\end{abstract}

\section{Introduction}

Self-reproduction or self-printing of programs is an old topic in programming. Self-reproductive program outputs the same source program as the original. For example, the following $\mathrm{C}$ program, which was programmed by Minoru Uehara, is a self-reproductive program [Ari 94].

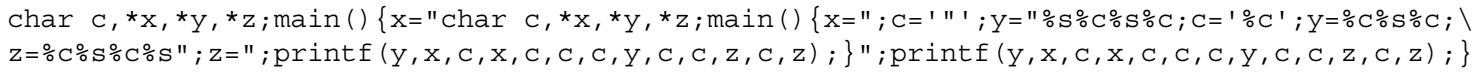

Self-reproduction of programs is probably useless in practical sense because nothing is changed by the reproduction.

This method can be applied to document reproduction, if a document can contain a program and it can reproduce a document that contains a program. An HTML page can contain a JavaScript program, and this program can clear the page content and generate new content when invoked. Thus, Web pages can be reproduced. The self-reproduction of a Web page is outlined in Figure 1. Whether a JavaScript program generated by the program works or not is not specified in JavaScript specification [Net 97a]. However, a self-reproductive page and the generated program partially but really works at least on Netscape Navigator 3.0 and 4.0! ${ }^{1}$ The mechanism of exact reproduction is explained more in Section 2.

Original HTML page
\begin{tabular}{|l|}
$<$ HTML $><$ HEAD $>$ \\
$<$ SCRIPT LANGUAGE= "JavaScript" \\
Self-reproducing \\
JavaScript Program \\
$</$ SCRIPT $>$ \\
$</$ HEAD $>$ \\
$<$ BODY $>$ \\
\hline DOCUment Content \\
$</$ BODY $>$ \\
$</$ HTML $>$ \\
\hline
\end{tabular}

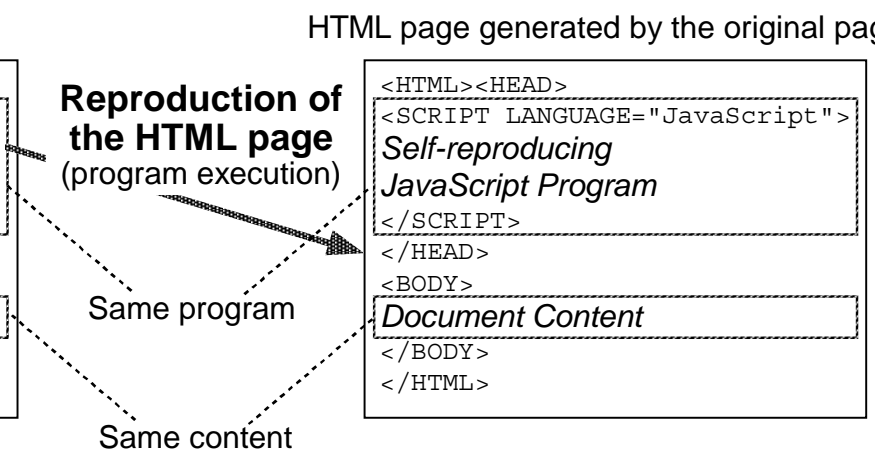

Figure 1. Outline of self-reproduction of Web pages

Exact reproduction of Web pages is probably useless in practical sense too, because of the same reason as the program reproduction. However, inexact reproduction may be useful because it can simulate modification or

${ }^{1}$ However, some examples do not work on Navigator 6.0 and 3.0. 
transformation of the document and the transformation may realize useful purposes. For example, inexact reproduction makes changing document view according to user's intention possible. The mechanism of document transformation using self-reproduction is explained in Section 3. Several examples, including a practical one, are shown in Section 4. The reason why whole reproduction of a Web page, instead of real partial rewriting, is necessary for document modification is that rewriting of original or previously generated text is not allowed in JavaScript. Rewriting is inhibited probably not because of an ad hoc reason, but because it causes serious semantic problem. There are other methods for changing document views, of course. However, the method using self-reproduction has several advantages, which are explained in Section 4.

We can understand a JavaScript program is reproduced and works on Navigators because its behavior is mostly as expected. However, it is difficult to look at the generated program itself using Navigators. A method for viewing generated program or method of debugging self-reproductive programs is explained in Section 5. Self-reproduction of programs seems to be tricky. A less tricky method for reproducing documents is overviewed in Section 6.

\section{Exact self-reproduction of documents}

A most simple example of exact self-reproduction of an HTML page is shown in Figure 2. This HTML page contains a button as its only visible content. The appearance of this page by Navigator is shown in Figure 3. The JavaScript program in Figure 2 is invoked when the user clicks the button shown in the window in Figure 3 . Then exactly the same HTML page is generated by the program and displayed on the window. Because the generated page is exactly the same, the appearance is the same as shown in Figure 3, and the user can repeat the reproduction again and again. This page is available at http://www.kanadas.com/reproduction/reproduction-b.html.

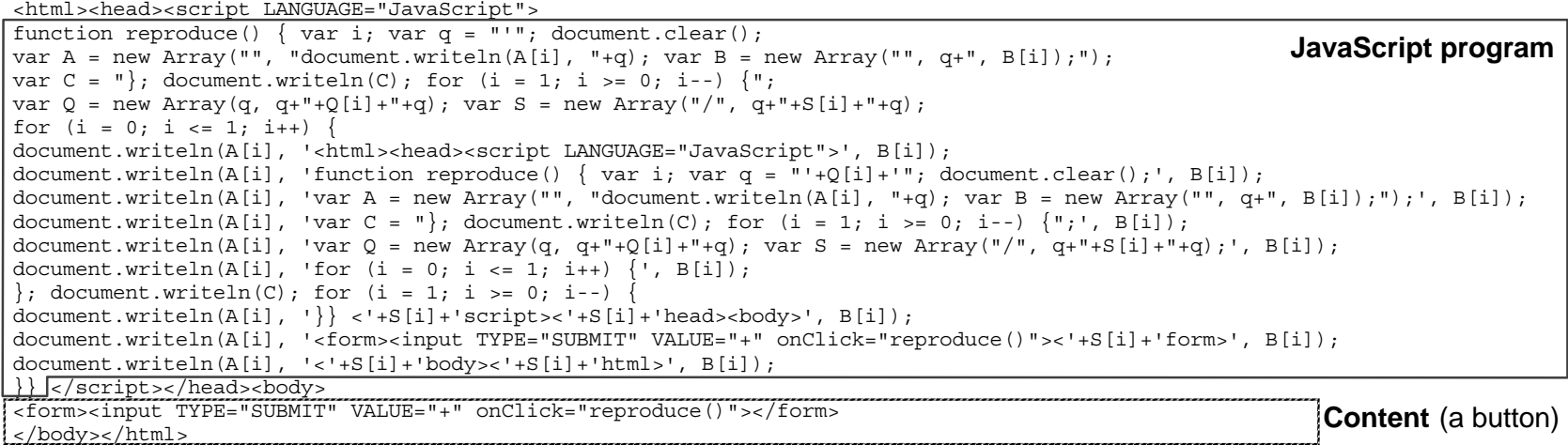

Figure 2. An exact self-reproductive page in HTML

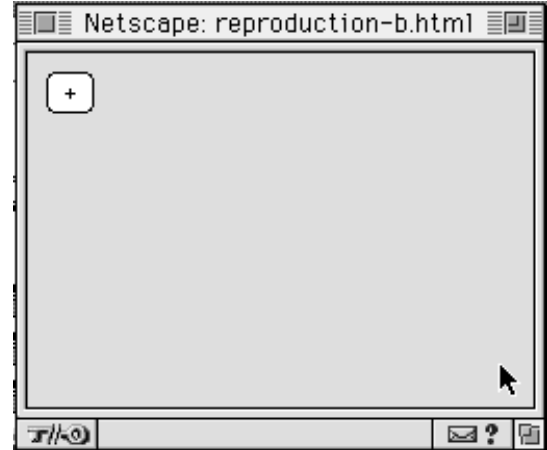

Figure 3. The appearance of the exact self-reproductive page ${ }^{1}$

The self-reproduction of this page really works on Navigator 3.0 or later versions, although not completely. It is not complete because, if the user repeats the reproduction process more than once, uses "go back" menu to back up to a

${ }^{1}$ Netscape Navigator on Apple Macintosh is used for showing the appearance of HTML pages thoughout this paper. 
reproduced page and clicks the button, then the button does not work as expected. It does not work because the program has gone! Microsoft Internet Explorer does not seem to reproduce a JavaScript program.

The process of self-reproduction is explained below. The same page as shown in Figure 2 is divided into five parts in Figure 4. The program in the first part, which is labeled "Part F1", erases the page content shown in Figure 4 (document.clear ();), and initializes arrays. Although the source of the running program is also erased, the running program itself, which is probably in compiled pseudo code, is (must be) preserved. The second part, which is labeled "Part F2," is part of the program, and it runs twice because it is in a for-statement. The same text as Part F1 is generated in the first run, and the same text as Part F2 is generated in the second run. The third part, Part C, runs once, and it generates the same text as Part C. The fourth part, Part L2, again runs twice. The same text as Part L2 is generated in the first run, and the same text as the last part, Part L1, is generated in the second run. Thus, the execution of whole program generates exactly the same text as the original HTML page.

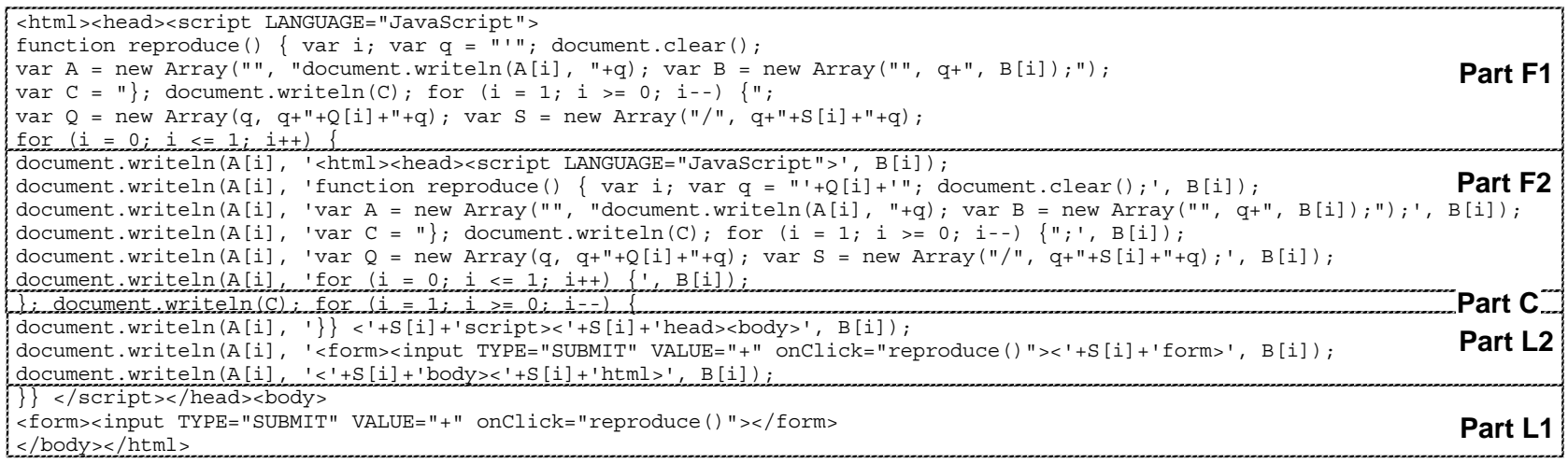

Figure 4. Divisions of the exact self-reproduction page

Arrays A and B are used for writing Part F1 and F2 in generated text differently. Array C is used for writing Part C. Arrays $Q$ and $S$ are used for writing single quote and slash characters. A quote or slash character must be written using a special technique because it requires an escape character when it is in a string.

\section{Partial transformation of documents by inexact self-reproduction}

Partial document transformation, i.e., generation of slightly different document page from the original, can be realized using a modified version of the reproductive page shown in Figure 2. A simple example, a page with a two-mode switch in a HTML page, is shown in Figure 5. The macroscopic structure of this page content is the same as the exact reproduction page. However, there are several small differences. This page contains a button labeled " + ". If the user clicks this button, a page that contains a button labeled "-" is generated. If the user clicks this new button, a page exactly the same as the original, i.e., the page that contains a button labeled "+", is generated. The user can repeat switching the button forever. The appearances of these two states are shown in Figure 6.

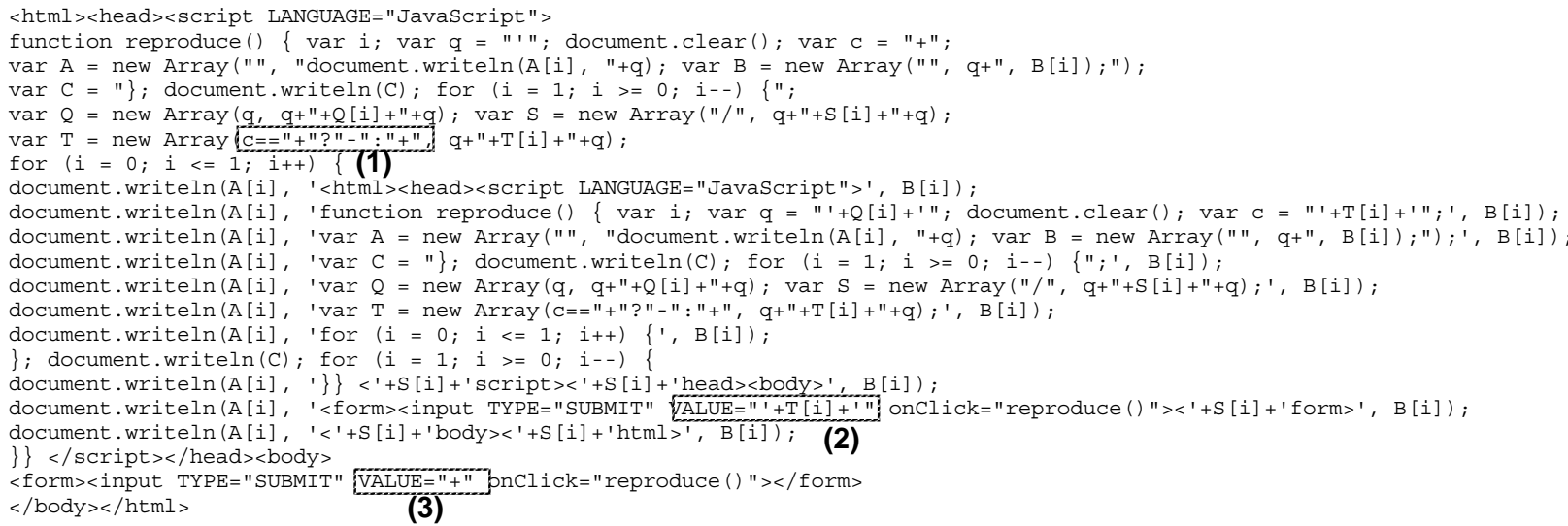

Figure 5. An inexact self-reproduction: a page with a switch 


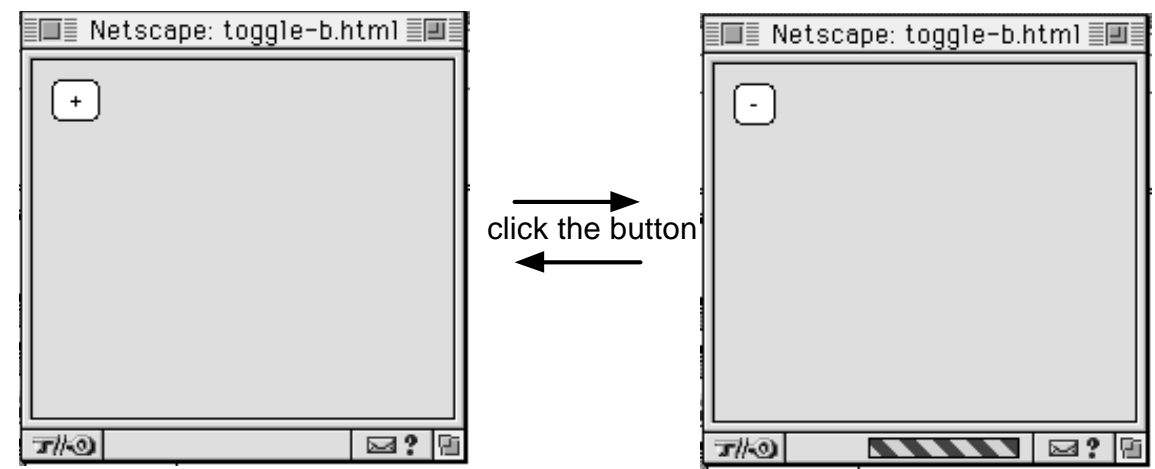

Figure 6. The appearance of two states of the page with a switch

Switching is realized by the following mechanism. The value of an expression labeled "(1)" in Figure 5 is "-” when the value of variable $\mathrm{C}$ is " + ", and it is "+" when the value of $\mathrm{C}$ is "-.". This value is stored into T[1], the second array element, by the assignment to T, and this value is used in the part labeled "(2)". The statement that contains this part runs twice. It generates the same text as itself in the first run, and, in the second run, it generates a button with "VALUE="-"," which is different from the part labeled "(3)" in the original page. This page is available at http://www.kanadas.com/reproduction/toggle-b.html.

\section{Applications of self-reproductive documents}

Several simple examples and a more practical example are presented in this section. All the examples are available at http://www.kanadas.com/reproduction/examples.html.

The first example, which is shown in Figure 7, is a page with a counter. The button in the original page is labeled "0." Every time the user clicks the button, the label is incremented: "1", "2", "3", and so on. Thus, the content of this page is continually changing and never becomes the same as an ancestor.

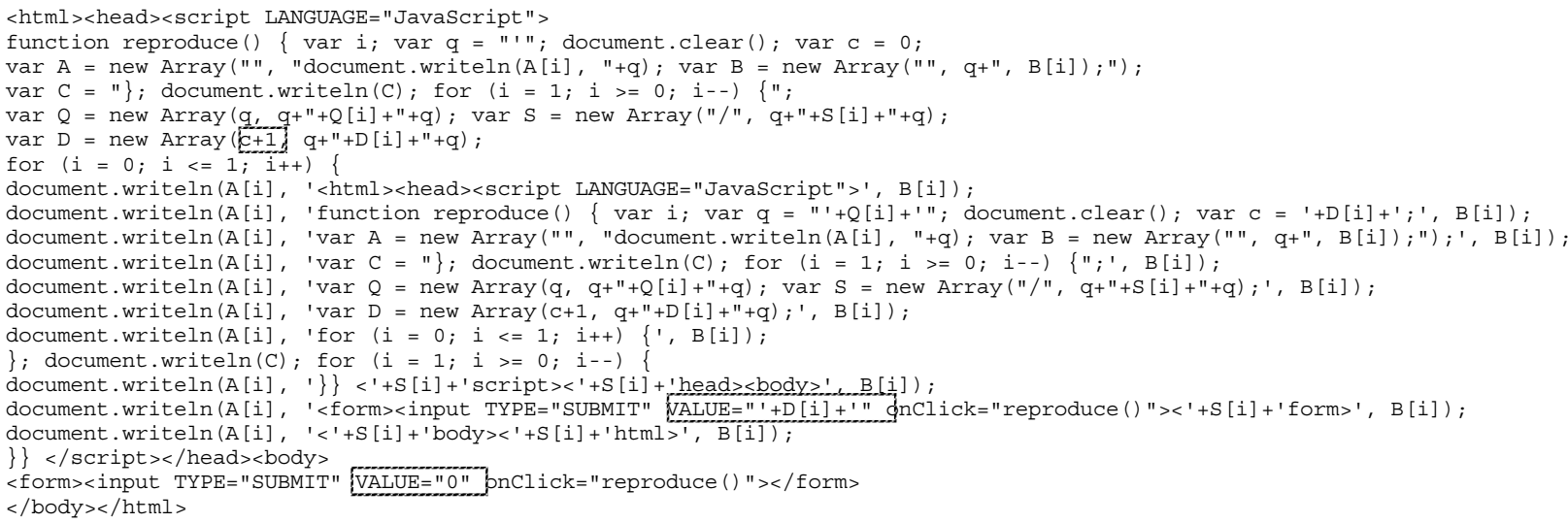

Figure 7. An inexact self-reproduction: a page with a counter

The second example, which is shown in Figure 8, is a page with simulated radio buttons. There are three buttons in this page. Only one of them is labeled "+" and the other two are labeled "-". The label of the button that the user clicks becomes "+", and others become "_".

The third example, which is shown in Figure 9, is more practical. There are three buttons and texts that accompany them. The appearance of this page is shown in Figure 10. The user can "open" or "close" the text that accompanies the button. "Closed" content may be an outline and opened content may be a more detailed content. This function is similar to the function of opening or closing folders in Explorer in Microsoft Windows 95 or in Finder in Apple Macintosh. Although the example shown in Figure 9 is still a toy, this function can be used for practical purposes if Web browsers support this function. 
<html ><head ><script LANGUAGE="JavaScript" >

function reproduce $(N) \quad$ (var $i$; var $q="$ '"; document.clear();

$\operatorname{var} \mathrm{A}=$ new Array (" ", "document. writeln $(\mathrm{A}[\mathrm{i}], "+\mathrm{q}) ; \operatorname{var} \mathrm{B}=$ new Array $("$ ", $q+", \mathrm{~B}[i]) ; ") ;$

var $C="\}$; document. writeln $(C)$; for $(i=1 ; i>=0 ; i--)\{" ;$

$\operatorname{var} Q=$ new $\operatorname{Array}(q, q+"+Q[i]+"+q) ; \operatorname{var} S=$ new $\operatorname{Array}(" / ", q+"+S[i]+"+q)$

var $\mathrm{TO}=$ new Array $(\mathrm{N}==0$ ? "+" : " $-", \mathrm{q}+"+\mathrm{T} 0[\mathrm{i}]+"+\mathrm{q})$;

$\operatorname{var} \mathrm{T} 1=$ new Array $(\mathrm{N}==1 ? "+": "-", \mathrm{q}+"+\mathrm{T} 1[\mathrm{i}]+"+\mathrm{q})$;

$\operatorname{var} \mathrm{T} 2=$ new Array $(\mathrm{N}==2 ? "+" \cdot n "-") \mathrm{q}+"+\mathrm{T} 2[\mathrm{i}]+"+\mathrm{q}){ }_{i}$

for $(i=0 ; i<=1 ; i++)$

document. writeln (A[i], '<html ><head><script LANGUAGE="JavaScript" > ', B [i]) ;

document.writeln(A[i], 'function reproduce $(N) \quad\{\operatorname{var} i$; var $q=" '+Q[i]+' " ;$ document.clear(); ', $B[i])$;

document.writeln(A[i], 'var $A=$ new Array("", "document.writeln(A[i], "+q); var B = new Array("", q+", B[i]);"); ', B[i]);

document.writeln $(A[i], ' \operatorname{var} C="\}$; document.writeln $(C)$; for $(i=1 ; i>=0 ; i--)\{" ; ', B[i])$;

document.writeln(A[i], $\operatorname{var} Q=$ new $\operatorname{Array}(q, q+"+Q[i]+"+q) ; \operatorname{var} S=$ new $\operatorname{Array}(" / ", q+"+S[i]+"+q) ; ', B[i])$;

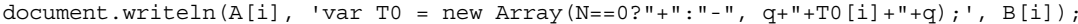

document. writeln (A[i], 'var $\mathrm{T} 1=\operatorname{new} \operatorname{Array}(\mathrm{N}==1$ ? "+" : " -", $\left.\mathrm{q}+"+\mathrm{T} 1[\mathrm{i}]+"+\mathrm{q}) ;{ }^{\prime}, \mathrm{B}[\mathrm{i}]\right)$;

document.writeln(A[i], 'var $\mathrm{T} 2=\operatorname{new} \operatorname{Array}(\mathrm{N}==2$ ? "+" : "-", $\left.\mathrm{q}+"+\mathrm{T} 2[\mathrm{i}]+"+\mathrm{q}) ;{ }^{\prime}, \mathrm{B}[\mathrm{i}]\right)$;

document.writeln(A[i], 'for $(i=0 ; i<=1 ; i++)\{1, B[i])$;

\}; document.writeln $(C)$; for $(i=1 ; i>=0$; $i--)$;

document.writeln $(A[i], 1\}\}<'+S[i]+$ 'script $><'+S[i]+$ 'head $><$ body $><$ form $>$ ', B [i]).

document, writeln (A[i], '<input TYPE="SUBMIT"

document.writeln(A[i], '<input TYPE="SUBMIT" YALUE=" '+T1 [i] +'" onclick="reproduce(1) "> ', B [i]) ;

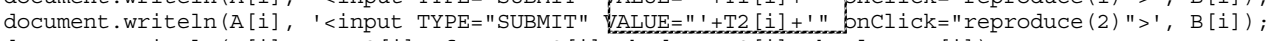

document.writeln (A[i], '<'+S[i]+' form $><$ '+S [i]+'body $><$ '+S[i]+'html ', B [i]);

\} $\}</$ script $></$ head $><$ body $><$ form $>$

$<$ input TYPE="SUBMIT" VALUE="+" pnClick="reproduce (p) $">$

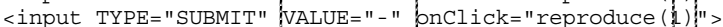

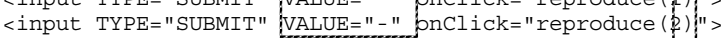

$</$ form $></$ body $></$ html $>$

\section{Figure 8. An inexact self-reproduction: a page with radio buttons}

$<$ html $><$ head $><$ script LANGUAGE= "JavaScript " >var $q="$ " "

function mkt $(N, V)$ \{ var $j$; this.length $=V$.length

for $(j=0 ; j<v$. length $; j++)\{\operatorname{var}$ not $=$ new Array $(0) ;$

not $["+"]="-" ; \operatorname{not}["-"]="+" ; \operatorname{not}[" \mathrm{closed} "]=$ "open"; not ["open"] = "closed";

this $[j]=$ new Array (Math.floor $(j / 2)==N$ ?not $[V[j]]: V[j], q+"+T["+j+"][i]+"+q) ;\}\}$

function reproduce $(\mathrm{N})$ \{ var $i$; document.clear ();

$\operatorname{var} \mathrm{A}=$ new Array("", "document.writeln(A[i], "+q); $\operatorname{var} \mathrm{B}=$ new Array("", q+", B[i]);");

$\operatorname{var} C="\}$; document.writeln $(C)$; for $(i=1 ; i>=0 ; i--)\{"$;

$\operatorname{var} Q=$ new $\operatorname{Array}(q, q+"+Q[i]+"+q) ; \operatorname{var} S=$ new $\operatorname{Array}(" / ", q+"+S[i]+"+q)$

$\operatorname{var} \mathrm{T}=$ new mkt $(\mathrm{N}$, new Array $("+"$, "closed",

"+", "closed", "+", "closed"));

for $(i=0 ; i<=1 ; i++)\{$

document. writeln (A[i], '<html ><head><script LANGUAGE="JavaScript" >var $q="$ '+Q [i] +'"; ', $B[i])$;

document.writeln (A[i], 'function $\operatorname{mkt}(\mathrm{N}, \mathrm{V})\{\operatorname{var} j ;$ this.length $=$ V.length; ', $\mathrm{B}[\mathrm{i}])$;

document. writeln(A[i], 'for $(j=0 ; j<V$.length $; j++)\{\operatorname{var}$ not $=$ new $\operatorname{Array}(0) ; 1, B[i]) ;$

document.writeln(A[i], 'not ["+"] = "-"; not ["-"] = "+"; not ["closed"] = "open"; not ["open"] = "closed"; , B [i]) ;

document.writeln (A[i], 'this $[j]=$ new Array (Math.floor $(j / 2)==N$ ? not $[V[j]]: V[j], q+"+T["+j+"][i]+"+q) ;\}\} ', B[i])$;

document.writeln(A[i], 'function reproduce $(\mathrm{N}) \quad$ var $i$; document.clear ()$\left._{i} ;, B[i]\right)$;

document.writeln(A[i], 'var $A=$ new Array("", "document.writeln(A[i], "+q); $\operatorname{var} B=\operatorname{new} A r r a y(" ", q+", B[i]) ; ") ; ', B[i])$;

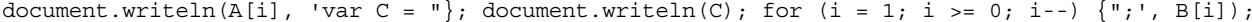

document. writeln $(A[i], \quad \operatorname{var} O=$ new $\operatorname{Array}(q, q+"+O[i]+"+q) ; \operatorname{var} S=\operatorname{new} \operatorname{Array}(" / ", q+"+S[i]+"+q) ; 1, B[i])$;

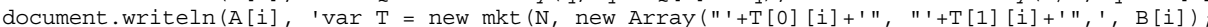

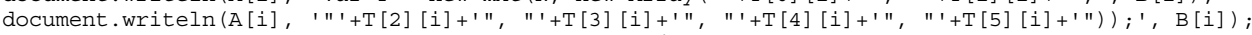

document.writeln(A[i], 'for $(i=0 ; i<=1 ; i++)\{1, B[i])$

\}; document.writeln $(C)$; for $(i=1 ; i>=0$; $i--)$

document.writeln $(A[i], '$ ' $\}\}<'+S[i]+$ 'script $><'+S[i]+$ 'head $><$ body $><$ pre $><$ form $>$ ', B [i]);

document. writeln (A[i], '<input TYPE="SUBMIT" VALUE=" '+T[0] [i] +'" onClick="reproduce (0) "> '+T[1] [i]+' ', B [i]) ;

document. writeln (A [i], '<input TYPE="SUBMIT" VALUE=" '+T[2] [i]+'" onClick="reproduce(1) ">'+T[3][i]+' ', B [i]),

document.writeln(A[i], '<input TYPE="SUBMIT" VALUE=" '+T[4][i]+'" onClick="reproduce(2) "> '+T[5] [i] +' ', B [i]);

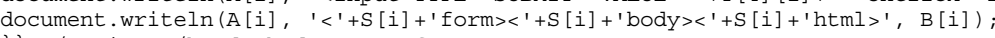

\}) </script $></$ head $><$ body $><$ pre $><$ form $>$

$<$ input TYPE="SUBMIT" VALUE=" +" onClick="reproduce (0) ">closed

<input TYPE="SUBMIT" VALUE="+" onClick="reproduce(1) ">closed

<input TYPE="SUBMIT" VALUE="+" onClick="reproduce (2) ">closed

$</$ form $></$ body $></$ html $>$

\section{Figure 9. An inexact self-reproduction: view change}

Document view change can be implemented using other methods such as below. However, the method using selfreproduction has several advantages as explained below.

- A method using a CGI scripts

In this method, when the user pushes a button on the page, a request is sent to a server using common gateway interface (CGI). The result is sent from the server to the client. The view can be changed very flexibly in this method and the programming a CGI script is relatively easy. However, the request and the changed page is sent through the network every time the user wants to change the view. Thus, the view change is much slower than the method using selfreproduction. 


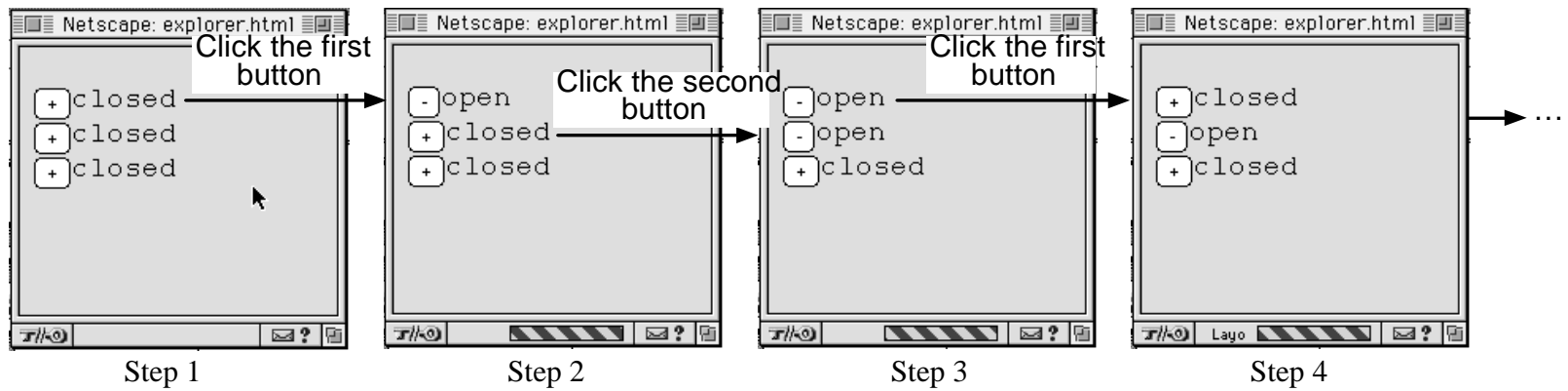

Figure 10. The appearance of the view change example page

- A method using Java or ActiveX

The buttons are placed on a Java applet [Sun 97] or ActiveX [Mic 97] control in this method. When the user pushes a button, the applet or control changes the view. The applet or control must control everything in the view. This means that it must implement a document browser. Thus, a large number of program steps is necessary and it is hard to make the new browser compatible with the original HTML browser. On the contrary, the original browser is used for displaying a reproduced text in the method using self-reproduction.

- A method using Dynamic HTML

View change can be implemented much easier by using Dynamic HTML [Net 97b] than using Java or ActiveX. Components of a page, which is called layers, can be added to or removed from the page dynamically. However, this method is still less flexible than the method using CGI or JavaScript, because displayed shapes of layers are limited to be rectangles, and the coordinates in the document must be specified in the page.

\section{A method for viewing generated programs}

We can recognize self-reproductive page works on Navigators because its behavior is mostly as expected. However, it is difficult to see the generated program itself using Navigators 3.0 or 4.0. If you open the page source window of Navigator when a generated HTML page is displayed, you will see the original page. If you go back to a generated page from another generated page and open the page source window, you can see the generated text. However, the source does not contain the program because of a bug of Navigators. Thus, another method for viewing generated programs is needed, especially when debugging a self-reproductive program.

A method for viewing generated programs is explained here. If you replace / head and script tag in the original page by body and pre tags as described below, this page generates visible text that is mostly the same as the page to be reproduced.

$</$ head $><$ script LANGUAGE="JavaScript" $>==><$ body $><$ pre $>$

However, this page is not reproductive any longer. If you perform the following process, which is shown in Figure 11, you will see the text. You click a button in the transformed page, click any button in the newly generated page, close all the JavaScript error message windows that have been opened by the mouse clicking, back up to the generated page, and open the document source window.

The above tag replacement makes the HTML page syntactically incorrect. If you hate syntax errors very much, you have to replace more tags. However, the above modification is enough for temporary purpose, i.e., debugging. 


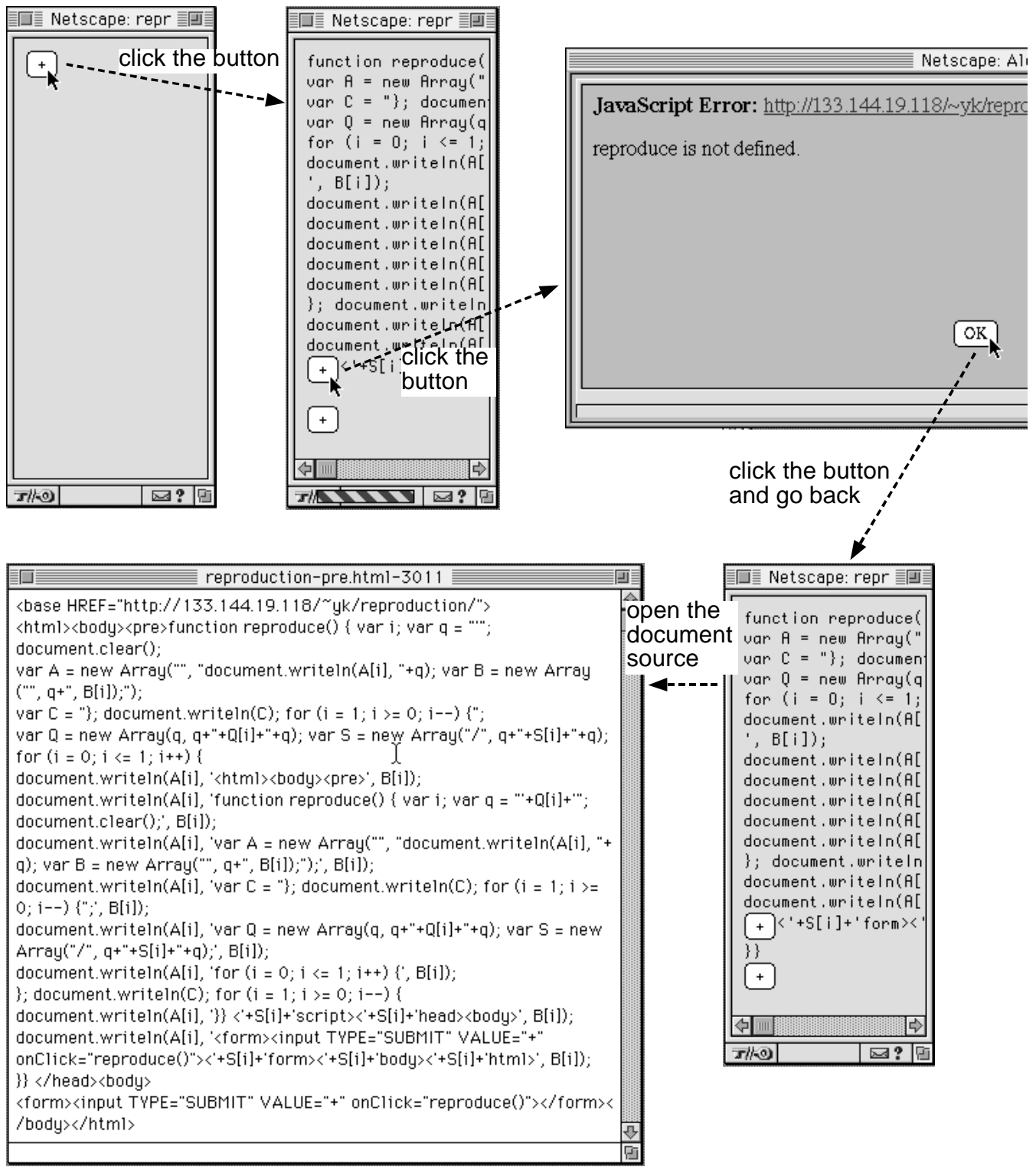

Figure 11. A method of viewing a generated page using Netscape Navigator 3.0

\section{Another method for reproducing documents}

Self-reproduction of programs seems to be tricky. There is a method for reproducing documents without reproducing programs. JavaScript program can be put in a separate file (URL) for Netscape Navigators. An exact self-reproductive page using this method is shown in Figure 12 (a), and the JavaScript program for this page is shown in Figure 12 (b). The relative URL of this program is reproduction-e.js. A script tag with SRC attribute, which is in Figure 12 (a), refers the external JavaScript program. This program reproduces the HTML page. The new page refers the same program. Inexact reproduction examples, which have been shown above, can also be rewritten easily using this method. A counter is implemented in Figure 13. Inexact reproduction can be realized using parameters to the JavaScript function. The function is reproduction and the parameter is $\mathrm{c}$ in this example.

A less tricky, clearer and, thus, more practical HTML pages and programs are obtained using this method. However, this method may be less interesting, and there are two disadvantages. One disadvantage is that this method is less powerful because the same program is used every time the page is reproduced. The other disadvantage is that programs 
in Figures 12 and 13 do not work on Navigator 3.0. The only current browsers, which these programs work on, is Navigator 4.0 and its preview versions.

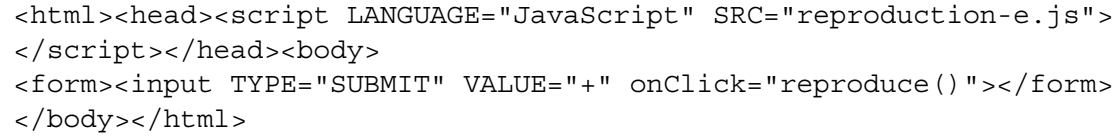

(a) The page content in HTML

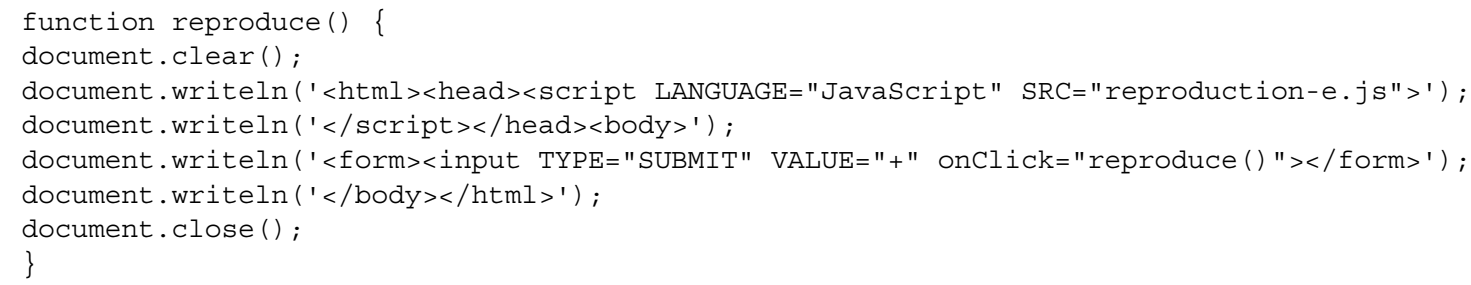

(b) The JavaScript program (reproduction-e.js)

\section{Figure 12. An exact self-reproduction without program reproduction}

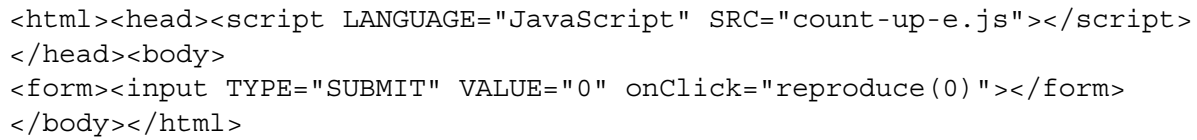

(a) The page content in HTML

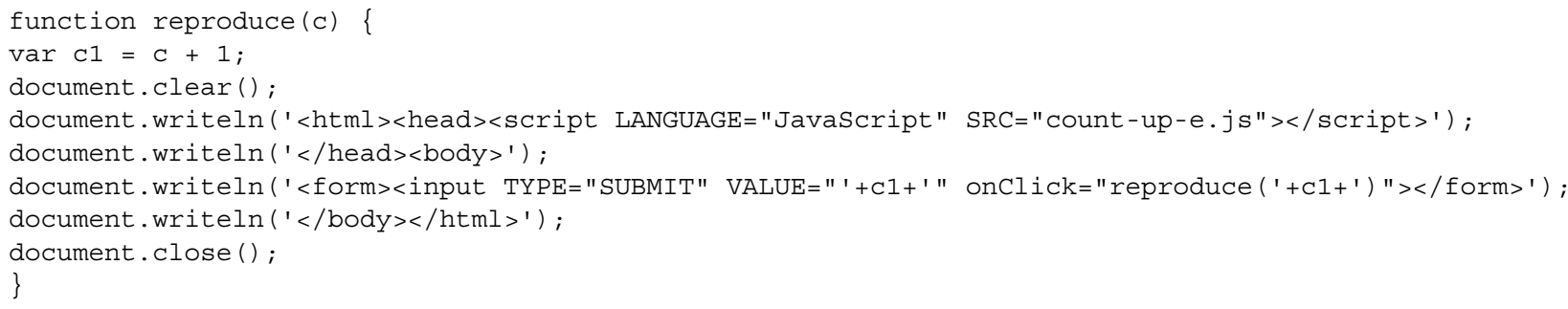

(b) The JavaScript program (count-up-e.js)

Figure 13. An inexact self-reproduction without program reproduction: a page with a counter

\section{Conclusion}

If a document can contain a program that reproduces the document, this function can be used for transforming the document. Changing the view or content of the document may be used for practical purposes, such as partially changing views from outline to detail. This function currently works on Netscape Navigators only partially. If future versions of Navigators, Internet Explorers and other Web browsers support reproduction function, this function can be really used throughout the Internet for changing document views and other purposes. The function can be implemented not only for HTML documents, but also for SGML or XML documents, and documents written in other mark-up languages.

\section{References}

[Ari 94] Arisawa, M.: Methods of Thinking in Programming, Nikkagiren Publisher, 1994 (in Japanese) [Mic 97] active platform, http://www.microsoft.com/activeplatform/default.asp, Microsoft Corporation.

[Net 97a] JavaScript 1.1 Language Specification, http://home.netscape.com/eng/javascript/index.html, Netscape Communications Corp.

[Net 97b] Netscape Communicator: What's Hot, http://home.netscape.com/comprod/products/communicator/beta_features.html, Netscape Communications Corp.

[Sun 97] What is Java?, http://java.sun.com/nav/whatis/index.html, Sun Microsystems, Inc. 